\title{
The fine geometry of the Cantor families of invariant tori in Hamiltonian systems ${ }^{\dagger}$
}

\author{
Àngel Jorba and Jordi Villanueva
}

\begin{abstract}
This work focuses on the dynamics around a partially elliptic, lower dimensional torus of a real analytic Hamiltonian system. More concretely, we investigate the abundance of invariant tori in the directions of the phase space corresponding to elliptic modes of the torus. Under suitable (but generic) nondegeneracy and non-resonance conditions, we show that there exists plenty of invariant tori in these elliptic directions, and that these tori are organized in manifolds that can be parametrized on suitable Cantor sets. These manifolds can be seen as "Cantor centre manifolds", obtained as the nonlinear continuation of any combination of elliptic linear modes of the torus. Moreover, for each family, the density of the complementary of the set filled up by these tori is exponentially small with respect to the distance to the initial torus. These results are valid in the limit cases when the initial torus is an equilibrium point or a maximal dimensional torus. It is remarkable that, in the case in which the initial torus is totally elliptic, we can derive Nekhoroshev-like estimates for the diffusion time around the torus. Due to the use of weaker non-resonance conditions, these results are an improvement on previous results [7].
\end{abstract}

\section{Introduction}

The study of the dynamics in a neighbourhood of a given invariant manifold is a classical topic in Dynamical Systems. Here we will focus on the description of the phase space around an invariant torus of a real analytic Hamiltonian system.

Let $H$ be a real analytic Hamiltonian system with $\ell$ degrees of freedom, having a $r$-dimensional invariant torus, $0 \leq r \leq \ell$. We assume that this torus is isotropic (the canonical symplectic form of $\mathbb{R}^{2 \ell}$ vanishes on the tangent bundle of the torus). Hence, there exists a set of canonical coordinates $(\theta, x, I, y) \in \mathbb{T}^{r} \times \mathbb{R}^{m} \times \mathbb{R}^{r} \times \mathbb{R}^{m}$, $m=\ell-r$, in such a way that the torus corresponds to the manifold $\{x=y=$ $0, I=0\}$, and it is parameterized by the angular variables $\theta$. Moreover, we will also assume that the dynamics on the torus is a linear quasi-periodic flow with respect to $\theta$, given by the vector of basic frequencies $\omega^{(0)} \in \mathbb{R}^{r}$. So, in these (canonical)

$\dagger$ Talk for the Third European Congress of Mathematics (Mini-symposium on Symplectic and Contact Geometry and Hamiltonian Dynamics). Barcelona, July 10th to 14th, 2000. 
coordinates the Hamiltonian can be written as

$$
H(\theta, x, I, y)=\left\langle\omega^{(0)}, I\right\rangle+\frac{1}{2}\langle z, B(\theta) z\rangle+H_{1}(\theta, x, I, y)
$$

where $z=(x, y)$ and the Taylor expansion of $H_{1}$, with respect to $(z, I)$, starts with monomials of degree 3 (as it is usual in this context, the degree of a monomial $z^{l} I^{s}, l \in \mathbb{N}^{2 m}, s \in \mathbb{N}^{r}$, is defined as $\left.\left|l_{1}+2\right| s\right|_{1}$, being $\left.|l|_{1}=\sum_{j}\left|l_{j}\right|\right)$.

As it is usual in KAM theory for lower dimensional tori, we will restrict to the context of the so-called reducible tori $([3])$. So, we will assume that the normal variational equations of the torus, which are given by $\dot{z}=J_{m} B\left(\omega^{(0)} t\right) z\left(J_{m}\right.$ denotes the matrix of the canonical 2 -form of $\left.\mathbb{R}^{2 m}\right)$, can be reduced to constant coefficients by means of a linear change of variables in $z$, depending on time in a quasi-periodic way (with $\omega^{(0)}$ as vector of basic frequencies). Then, we can perform a canonical transformation on the initial Hamiltonian system (depending on $\theta$ in a $2 \pi$-periodic way), such that (1) takes the form

$$
H(\theta, x, I, y)=\left\langle\omega^{(0)}, I\right\rangle+\frac{1}{2}\langle z, B z\rangle+H_{1}(\theta, x, I, y),
$$

where $B$ is a symmetric matrix with constants coefficients. To simplify the notations, here we have also kept the names $H$ and $H_{1}$ for the Hamilton function and for the higher order terms. Moreover, in order to have a Hamiltonian given by a real function, we restrict our set-up to the case in which the linear transformation is given by a real change of variables, and hence, we assume that we have a real reduced matrix $B$. The linear normal behavior around the torus is now given by the system $\dot{z}=J_{m} B z$. For definiteness, we denote the eigenvalues of $J_{m} B$ as $(\lambda,-\lambda)=\left(\lambda_{1}, \ldots, \lambda_{m},-\lambda_{1}, \ldots,-\lambda_{m}\right)$. As usual, we will call the imaginary part of these eigenvalues the normal frequencies of the torus.

We are concerned with the local behaviour around this lower dimensional torus. Let us start by looking at the linear approximation to this dynamics. As the dynamics in the hyperbolic directions (eigenvalues with nonzero real part) is locally very simple -trajectories either escape or tend asymptotically to the toruswe will focus on the elliptic directions. For simplicity, let us start by assuming that $\pm \lambda_{1}$ are a conjugate pair of purely imaginary eigenvalues, $\lambda_{1}=i \omega_{r+1}^{(0)}$. It is clear that the dynamics in the corresponding two-dimensional real invariant space is that of a linear oscillator, with frequency $\omega_{r+1}^{(0)}$. More concretely, this linear subspace is filled by a 1-parametric family of periodic orbits whose frequency is fixed, i.e., it does not depend on the amplitude. Hence, taking into account that these linear oscillations take place around a $r$-dimensional torus carrying a linear dynamics with frequency $\omega^{(0)}$, we have that the (linear approximation to the) dynamics in the (real) eigenspace corresponding to $\pm \lambda_{1}$ is described by a 1-parametric family of $(r+1)$-dimensional tori, having $\left(\omega^{(0)}, \omega_{r+1}^{(0)}\right)$ as a vector of basic frequencies. This family is degenerate, in the sense that the frequencies are constant. A natural question is the effect that the nonlinear terms have on this picture. We will show that, under generic conditions, this family "survives" the effect of the nonlinear terms, but it becomes Cantorian (and now, the frequencies 
depend on the amplitudes), see $[4,17,20,7,21]$. The dense set of holes that conform the Cantor structure is due to the resonances between the basic and normal frequencies. We will also show that the size of those holes is exponentially small with respect to the distance to the initial torus.

The above description has been done using a single normal frequency and a single $r$-dimensional torus. A similar discussion can be made using several normal frequencies: if we select a set of $m_{1}$ normal frequencies then, in the corresponding directions, the dynamics of the linear system is described by a $m_{1}$-parametric family of $m_{1}$-dimensional tori, having all of them the same frequencies. Moreover, if we skip the term $H_{1}$ in (2), it is clear that the initial torus is embedded in a $r$-parametric family of $r$-dimensional tori (parameterized by $I$ ), that also becomes Cantorian when we consider the nonlinear part (see $[4,17,3,7,21]$ ). Combining this family with the families in the normal directions we obtain a $\left(r+m_{1}\right)$ parametric family of $\left(r+m_{1}\right)$-dimensional tori. As we will see, most of these tori survive the effect of $H_{1}$, and are organized as Cantor manifolds. As before, the measure of the holes of the Cantor structure is exponentially small with respect to the distance to the initial torus.

In the next sections we will discuss these issues in more detail, and we will give some hints about the scheme used in the proofs (full details can be found in [7] and [9]). Moreover, in the case in which the torus is totally elliptic, we will derive lower bounds on the escaping time from a neighbourhood of the torus.

\section{Hypotheses and set-up}

We will assume that the Hamiltonian (2) is a real analytic function, defined on

$$
\mathcal{D}_{r, m}\left(\rho_{0}, R_{0}\right)=\left\{(\theta, x, I, y) \in \mathbb{C}^{2 \ell}:|\operatorname{Im}(\theta)| \leq \rho_{0},|z| \leq R_{0},|I| \leq R_{0}^{2}\right\},
$$

for certain $0<\rho_{0}<1$ and $0<R_{0}<1$, where $|\cdot|$ refers to the sup norm of complex vectors. We will denote by $\|\cdot\|_{\rho, R}$ the sup norm of a function on the set $\mathcal{D}_{r, m}(\rho, R)$ and we will also assume that $\|H\|_{\rho_{0}, R_{0}}<+\infty$.

As we are interested in the dynamics in the elliptic directions of the torus, let us define $m_{e}$ as the number of elliptic directions of the initial torus, and let us write $\lambda=\left(\lambda^{e}, \lambda^{h}\right)$, where $\pm \lambda^{e}$ denotes the $2 m_{e}$ elliptic eigenvalues, and $\pm \lambda^{h}$ the $2 m_{h}$ hyperbolic ones $\left(m=m_{e}+m_{h}\right)$. Let $m_{1}$ be any number of elliptic directions $\left(0 \leq m_{1} \leq m_{e}\right)$ that, without loss of generality, we will assume that correspond to the first $m_{1}$ elliptic eigenvalues. So, we will write $\lambda^{e}=\left(\widetilde{\lambda}^{e}, \widehat{\lambda}^{e}\right)$, where $\widetilde{\lambda}^{e}$ are these first $m_{1}$ components and $\hat{\lambda}^{e}$ the remaining ones. Moreover, we will denote $\widetilde{\lambda}^{e}=i \widetilde{\omega}^{(0)}$, and $\Omega^{(0)}=\left(\omega^{(0)}, \widetilde{\omega}^{(0)}\right) \in \mathbb{R}^{r+m_{1}}$. We want to construct the family of invariant tori for the elliptic directions corresponding to the frequency $\widetilde{\omega}^{(0)}$ (so, the basic frequencies of this family will be close to $\left.\Omega^{(0)}\right)$. These tori will have $m_{e}-m_{1}$ elliptic directions and $m_{h}$ hyperbolic ones. 


\subsection{Non-resonance conditions}

An important point for the quantitative analysis is the control of the small divisors. So, we will assume that some linear combinations of basic frequencies and normal eigenvalues satisfy a standard Diophantine condition. More concretely, we suppose that, for certain $c>0$ and $\gamma>r+m_{1}-1$, we have

$$
\left|i\left\langle k, \Omega^{(0)}\right\rangle+\left\langle\widehat{\lambda}^{e}, \widehat{l^{e}}\right\rangle\right| \geq \frac{c}{\left(|k|_{1}+|\widehat{l}|_{1}\right)^{\gamma}},
$$

for any $k \in \mathbb{Z}^{r+m_{1}}$ and any $\widehat{l^{e}} \in \mathbb{Z}^{m_{e}-m_{1}}$, with $|\widehat{l}|_{1} \leq 2,|k|_{1}+\mid \widehat{l}{ }^{e}{ }_{1} \neq 0$. It is well known that if we take a fixed $\gamma>r+m_{1}-1$, the set of $\left(\Omega^{(0)}, \hat{\lambda}^{e}\right)$ for which these conditions are not fulfilled, for any $c>0$, has zero measure.

\section{Normal form}

This is the first step of the methodology used. The purpose is to apply a (canonical) change of variables to bring Hamiltonian (2) into a more convenient form. So, we will perform a finite number of normal form transformations, in order to kill some specific monomials of the Taylor-Fourier expansion of the Hamiltonian. In order to enumerate these monomials, we introduce the following notation. Let us split $z$ as $\left(z^{e}, z^{h}\right)$, where $z^{e}$ denotes the variables corresponding to the elliptic normal directions, and $z^{h}$ to the hyperbolic ones. Moreover, we write $z^{e}=\left(\widetilde{z}^{e}, \widehat{z}^{e}\right)$, where $\widetilde{z}^{e}$ refers to the variables related to the first $m_{1}$ elliptic directions (the corresponding frequencies are $\left.\widetilde{\omega}^{(0)}\right)$, and $\widehat{z}^{e}$ refers to the remaining elliptic directions. Finally, $z^{n}$ is defined as $\left(\widehat{z}^{e}, z^{h}\right)\left(z^{n}\right.$ will be the normal directions to the new family of invariant tori). Hence, the monomials we would like to remove are:

1. all the monomials of order one in $z^{n}$; then, $z^{n}=0$ is an invariant manifold,

2. all the monomials that do not contain $z^{n}$ (except the unavoidable resonances); then, the manifold $z^{n}=0$ is foliated by invariant tori,

3. all the monomials of order two in $z^{n}$ that involve either one elliptic and one hyperbolic direction or two elliptic directions (except, of course, the unavoidable resonances); then, the elliptic part of the normal variational equation of these tori is reduced to constant coefficients.

As it is well known, killing these monomials at all orders is a (generically) divergent process, so we will remove them up to a suitable finite degree. This degree will be selected depending on the distance to the initial torus, in order to have an exponentially small remainder with respect to that distance (see Theorem 3.1). To write this normal form, let $\widetilde{I}_{j}$ be $\frac{1}{2}\left(\left(x_{j}^{e}\right)^{2}+\left(y_{j}^{e}\right)^{2}\right)\left(j=1, \ldots, m_{1}\right)$, and $\bar{I}=(I, \widetilde{I})$. Then, skipping the higher order terms that do not belong to the normal form, we have that

$$
H(\theta, x, I, y)=F(\bar{I})+\frac{1}{2}\left\langle z^{n}, Q^{n}\left(\theta, I, \widetilde{z}^{e}\right) z^{n}\right\rangle+O_{3}\left(z^{n}\right),
$$


where the matrix $Q^{n}$ takes the following form:

$$
\left\langle z^{n}, Q^{n}\left(\theta, I, \widetilde{z}^{e}\right) z^{n}\right\rangle=\left\langle\hat{z}^{e}, Q^{e}(I) \hat{z}^{e}\right\rangle+\left\langle z^{h}, Q^{h}\left(\theta, I, \tilde{z}^{e}\right) z^{h}\right\rangle
$$

and $\partial_{\bar{I}} F(0)=\Omega^{(0)}$. The action $\widetilde{I}$ can be taken as a parameter in the normal direction. It is easy to check that we have the following quasi-periodic solutions for the canonical equations associated to (5):

$$
\begin{aligned}
\theta(t) & =\partial_{I} F\left(\bar{I}^{(0)}\right) t+\theta^{(0)}, \\
I(t) & =I^{(0)}, \\
\widetilde{x}_{j}^{e}(t) & =\sqrt{2 \widetilde{I}_{j}^{(0)}} \sin \left(\partial_{\widetilde{I}_{j}} F\left(\bar{I}^{(0)}\right) t+\widetilde{\theta}_{j}^{(0)}\right), \\
\widetilde{y}_{j}^{e}(t) & =\sqrt{2 \widetilde{I}_{j}^{(0)}} \cos \left(\partial_{\widetilde{I}_{j}} F\left(\bar{I}^{(0)}\right) t+\widetilde{\theta}_{j}^{(0)}\right), \\
z^{n}(t) & =0,
\end{aligned}
$$

where $\bar{I}^{(0)}=\left(I^{(0)}, \widetilde{I}^{(0)}\right)$ and $\widetilde{\theta}_{j}^{(0)}$ are arbitrary initial conditions. The following result contains the estimates for the normal form.

Theorem 3.1. For any $R>0$ small enough, there exists a real analytical canonical transformation $\Psi^{R}: \mathcal{D}_{r, m}\left(\rho_{0} / 2, R \mathrm{e}^{-\rho_{0} / 2}\right) \mapsto \mathcal{D}_{r, m}\left(\rho_{0}, R\right)$, with $\Psi^{R}-I d 2 \pi$-periodic in $\theta$ and small. More concretely, if we write $\Psi^{R}-I d=\left(\Theta^{R}, \mathcal{X}^{R}, \mathcal{I}^{R}, \mathcal{Y}^{R}\right)$, and $\mathcal{Z}^{R}=\left(\mathcal{X}^{R}, \mathcal{Y}^{R}\right)$, we have

$$
\left\|\Theta^{R}\right\| \leq \rho_{0} / 2, \quad\left\|\mathcal{I}^{R}\right\| \leq R^{2}\left(1-\mathrm{e}^{-4 \rho_{0}}\right), \quad\left\|\mathcal{Z}^{R}\right\| \leq R\left(1-\mathrm{e}^{-2 \rho_{0}}\right) .
$$

The transformed Hamiltonian $H^{R}=H \circ \Psi^{R}$ takes the form

$$
H^{R}(\theta, x, I, y)=F^{R}(\bar{I})+\frac{1}{2}\left\langle z^{n}, Q^{R, n}\left(\theta, I, \tilde{z}^{e}\right) z^{n}\right\rangle+S^{R}(\theta, x, I, y)+T^{R}(\theta, x, I, y),
$$

where, skipping the term $S^{R}$, we have the normal form (5), with $T^{R} \equiv O_{3}\left(z^{n}\right)$. Moreover, we have the following estimate:

$$
\left\|S^{R}\right\| \leq c_{1} \mathrm{e}^{-\left(c_{1} / R\right)^{\frac{2}{\gamma+1}}}
$$

where $c_{1}>0$ is a constant. All the norms are taken on the set $\mathcal{D}_{r, m}\left(\rho_{0} / 2, R \mathrm{e}^{-\rho_{0} / 2}\right)$.

We point out that the kind of dependence of $\Psi^{R}$ on $R$ does not matter for our purposes. What follows from the proof is that it is piecewise analytic.

Remark 3.2. If the torus is totally elliptic $\left(m_{h}=0\right)$, the bound on the remainder $S^{R}$ immediately implies a Nekhoroshev estimate on the diffusion time around the torus (see $[14,16,12,7,15])$. More concretely, it can be shown that the time needed to go from a distance of $R$ to a distance of $2 R$ has to be bigger than $c_{2} \exp \left(\left(c_{2} / R\right)^{2 /(\gamma+1)}\right)$, for some constant $c_{2}>0$. This includes the limit cases of elliptic equilibrium points as well as of maximal dimensional tori. 


\section{Cantor manifolds of lower dimensional tori}

The usual schemes to prove the existence of invariant tori are based on an iterative process with quadratic convergence (Newton method), to overcome the effect of the small divisors $([10,1])$. These methods are usually constructed by means of a sequence of canonical transformations, that puts the initial Hamiltonian into a form where the existence of the tori follows immediately.

The equations that define the canonical transformations used at each step of the Newton method are given by a linear system of partial differential equations. In order to simplify the resolution of these equation we only deal with tori that are reducible in the elliptic directions: in this way, we directly solve this part of the equations by reducing them to constant coefficients while the hyperbolic part can be easily solved by means of a standard fixed point scheme ([5]). The reducibility has been imposed in the construction of the normal form in Section 3 and in the Diophantine condition (4) by asking for $\left|\hat{l}^{e}\right|_{1}=2$. Following the ideas in [2], it should be possible to construct invariant tori without asking for reducibility, that is, only asking for $\left|\hat{l}^{e}\right|_{1} \leq 1$ in (4).

As it is usual in these situations, we need to control the frequencies along the iterative process, to avoid divisors that are too small. To this end we will use suitable non-degeneracy conditions to control the dependence of the frequencies with respect to a suitable set of parameters. The internal frequencies of the tori in the family can be easily controlled by the corresponding actions $\bar{I}$ (see hypothesis $\mathbf{H 1}$ in Theorem 4.1). The control of the frequencies in the elliptic directions is more involved, since we do not have free parameters available (this constitutes the so-called lack-of-parameters problem, $[13,6,22,3,8])$. Here we also use the actions $\bar{I}$, by asking for a suitable transversality condition with respect to the set of resonant frequencies (see hypothesis $\mathbf{H 2}$ in Theorem 4.1).

Note that the lower bound (4) for the small divisors appears in the denominators of the coefficients of the series involved in the Newton process. As this iterative process will be applied to the Hamiltonian obtained from Theorem 3.1, the remainder to be killed is exponentially small. Then, we can select the value of $c$ in (4) to be of the same order as this remainder, such that the measure of the frequencies that do not satisfy (4) is also exponentially small. Then, the abovementioned non-degeneracy conditions allows to complete the proof, except for a set of frequencies of exponentially small measure.

Theorem 4.1. Assume the same hypotheses as in Theorem 3.1, and compute the normal form (5) up to degree 2. Let us define $\mu(\bar{I})$ as the normal frequencies of the elliptic directions of the family of lower dimensional tori in this normal form. Then, we assume the following non-degeneracy conditions:

H1: $\operatorname{det} \partial_{\bar{I}, \bar{I}}^{2} F(0) \neq 0$,

H2: $\operatorname{Re}\left(l^{\top} \partial_{\bar{I}} \mu(0)\left(\partial_{\bar{I}, \bar{I}}^{2} F(0)\right)^{-1}\right) \notin \mathbb{Z}^{r+m_{1}}$, for any $l \in \mathbb{Z}^{m_{e}-m_{1}}, 0<|l|_{1} \leq 2$.

Then, there exists $c_{0}>0$ such that for any $R>0$ small enough, there is a Cantor set $\mathcal{W}_{R} \subset \mathcal{V}_{R}=\left\{\Omega \in \mathbb{R}^{r+m_{1}}:\left|\Omega-\Omega^{(0)}\right| \leq c_{0} R^{2}\right\}$ such that, for any $\Omega \in \mathcal{W}_{R}$, 
the Hamiltonian system $H$ has an invariant torus of dimension $r+m_{1}$, having $\Omega$ as vector of basic frequencies, that is close to the one predicted by the normal form. The elliptic directions of the normal variational equations of these tori are reducible. Moreover, $\mathcal{W}_{R}$ can be characterized in terms of the Lebesgue measure:

$$
\operatorname{meas}\left(\mathcal{V}_{R} \backslash \mathcal{W}_{R}\right) \leq c_{0} \mathrm{e}^{-\left(c_{0} / R\right)^{\frac{2}{\gamma+1}}}
$$

Conditions $\mathbf{H 2}$ are usually called first order Melnikov conditions (see $[11,4]$ ).

Remark 4.2. Condition $\mathrm{H1}$ is a standard (and generic) non-degeneracy condition on the dependence of the basic frequencies on the actions $([10,1])$. A similar result can be proved replacing $\mathbf{H 1}$ by a higher order non-degeneracy condition $([18,19])$.

Remark 4.3. It can also be proved that the invariant Cantor manifold can be extended to a (non invariant) $C^{\infty}$ manifold that contains all the invariant tori in the family (see [23]). The measure of the complementary of the set of tori on this manifold is also exponentially small.

Remark 4.4. Some of the invariant tori provided by Theorem 4.1 are "complex tori", in the sense that the corresponding quasi-periodic trajectories (for real time) live in $\mathbb{C}^{2 \ell}$. This follows immediately from (6) by selecting some $\widetilde{I}_{j}$ negative.

\section{Acknowledgements}

The authors are indebted with M. Sevryuk for his remarks. This research has been supported by the Spanish grant DGICYT PB94-0215, the Catalan grant CIRIT 1998S0GR-00042 and the INTAS project 97-10771.

\section{References}

[1] V.I. Arnold. Proof of A.N. Kolmogorov's theorem on the preservation of quasiperiodic motions under small perturbations of the Hamiltonian. Russian Math. Surveys, 18(5):9-36, 1963.

[2] J. Bourgain. On Melnikov's persistency problem. Math. Res. Lett., 4(4):445-458, 1997.

[3] H.W. Broer, G.B. Huitema, and M.B. Sevryuk. Quasi-Periodic Motions in Families of Dynamical Systems: Order amidst Chaos, volume 1645 of Lecture Notes in Math. Springer, New York, 1996.

[4] L.H. Eliasson. Perturbations of stable invariant tori for Hamiltonian systems. Ann. Sc. Norm. Super. Pisa, Cl. Sci., 15(1):115-147, 1988.

[5] S. Graff. On the conservation of hyperbolic invariant tori for Hamiltonian systems. J. Differential Equations, 15(1):1-69, 1974.

[6] À. Jorba and C. Simó. On the reducibility of linear differential equations with quasiperiodic coefficients. J. Differential Equations, 98:111-124, 1992.

[7] À. Jorba and J. Villanueva. On the normal behaviour of partially elliptic lower dimensional tori of Hamiltonian systems. Nonlinearity, 10:783-822, 1997. 
[8] À. Jorba and J. Villanueva. On the persistence of lower dimensional invariant tori under quasi-periodic perturbations. J. Nonlinear Sci., 7:427-473, 1997.

[9] À. Jorba and J. Villanueva. On the density of quasi-periodic motions around a partially elliptic lower dimensional torus. Preprint, 2000.

[10] A.N. Kolmogorov. On the persistence of conditionally periodic motions under a small change of the Hamilton function. Dokl. Acad. Nauk. SSSR, 98(4):527-530, 1954.

[11] V.K. Melnikov. On some cases of the conservation of conditionally periodic motions under a small change of the Hamiltonian function. Soviet Math. Dokl., 6:1592-1596, 1965.

[12] A. Morbidelli and A. Giorgilli. Superexponential stability of KAM tori. J. Statist. Phys., 78(5-6):1607-1617, 1995.

[13] J. Moser. Convergent series expansions for quasi-periodic motions. Math. Ann., 169:136-176, 1967.

[14] N.N. Nekhoroshev. An exponential estimate of the time of stability of nearlyintegrable Hamiltonian systems. Russian Math. Surveys, 32:1-65, 1977.

[15] L. Niederman. Nonlinear stability around an elliptic equilibrium point in a Hamiltonian system. Nonlinearity, 11(5):1465-1479, 1998.

[16] A.D. Perry and S. Wiggins. KAM tori are very sticky: rigorous lower bounds on the time to move away from an invariant Lagrangian torus with linear flow. Phys. D, 71:102-121, 1994.

[17] J. Pöschel. On elliptic lower dimensional tori in Hamiltonian systems. Math. Z., 202(4):559-608, 1989.

[18] H. Rüssmann. Non-degeneracy in the perturbation theory of integrable dynamical systems. In Number theory and dynamical systems, volume 134 of Lond. Math. Soc. Lect. Note Ser., pages 5-18. 1989.

[19] M.B. Sevryuk. Invariant tori of Hamiltonian systems that are nondegenerate in Rüssman's sense. Dokl. Akad. Nauk, Ross. Akad. Nauk, 346(5):590-593, 1996.

[20] M.B. Sevryuk. Excitation of elliptic normal modes of invariant tori in Hamiltonian systems. In A.G. Khovanskii, A.N. Varchenko, and V.A. Vassiliev, editors, Topics in Singularity Theory. V.I. Arnold's 60th Anniversary Collection, volume 180 of American Mathematical Society Translations-Series 2, Advances in the Mathematical Sciences, pages 209-218. American Mathematical Society, Providence, Rhode Island, 1997.

[21] M.B. Sevryuk. Invariant tori of intermediate dimensions in Hamiltonian systems. Regul. Chaotic Dyn., 3(1):39-48, 1998.

[22] M.B. Sevryuk. The lack-of-parameters problem in the KAM theory revisited. In C. Simó, editor, Hamiltonian Systems with Three or More Degrees of Freedom, NATO Adv. Sci. Inst. Ser. C Math. Phys. Sci., pages 568-572. Held in S'Agaró, Spain, 1930 June 1995. Kluwer Acad. Publ., Dordrecht, Holland, 1999.

[23] H. Whitney. Analytic extensions of differentiable functions defined in closed sets. Trans. Amer. Math. Soc., 36(1):63-89, 1934. 
À. Jorba:

Departament de Matemàtica Aplicada i Anàlisi, Universitat de Barcelona,

Gran Via 585, 08007 Barcelona, Spain.

E-mail address: angel@maia.ub.es

J. Villanueva:

Departament de Matemàtica Aplicada I, Universitat Politècnica de Catalunya,

Diagonal 647, 08028 Barcelona, Spain.

E-mail address: jordi@tere.upc.es 